University of Nebraska - Lincoln

DigitalCommons@University of Nebraska - Lincoln

2003

\title{
Estimated risk of transmission of the West Nile virus through blood transfusion in the US, 2002
}

\author{
Brad J. Biggerstaff \\ Centers for Disease Control and Prevention, bbiggerstaff@cdc.gov \\ Lyle R. Petersen \\ Centers for Disease Control and Prevention, Ipetersen@cdc.gov
}

Follow this and additional works at: https://digitalcommons.unl.edu/publichealthresources

Part of the Public Health Commons

Biggerstaff, Brad J. and Petersen, Lyle R., "Estimated risk of transmission of the West Nile virus through blood transfusion in the US, 2002" (2003). Public Health Resources. 235.

https://digitalcommons.unl.edu/publichealthresources/235

This Article is brought to you for free and open access by the Public Health Resources at DigitalCommons@University of Nebraska - Lincoln. It has been accepted for inclusion in Public Health Resources by an authorized administrator of DigitalCommons@University of Nebraska - Lincoln. 


\title{
Estimated risk of transmission of the West Nile virus through blood transfusion in the US, 2002
}

\author{
Brad J. Biggerstaff and Lyle R. Petersen
}

BACKGROUND: The West Nile virus (WNV) epidemic in 2002 in the US saw over 3300 reported human cases of WNV disease, with over 2300 reported cases of WNV encephalitis and meningitis. The first documented cases of transfusion transmission of WNV through voluntary blood donation also occurred. STUDY DESIGN AND METHODS: Case onset dates from the 2002 WNV epidemic in the US were used to estimate the risk of transfusion-associated transmission with statistical resampling. An easily computed approximating formula for the mean risk was derived. Estimates were computed for six high-incidence states and metropolitan areas.

RESULTS: Mean and maximum risk of transfusionassociated WNV transmission (per 10,000 donations) during the epidemic period for the selected states ranged from 2.12 to 4.76 and from 4.34 to 10.46 , respectively; for the selected metropolitan areas, they ranged from 1.46 to 12.33 and from 3.02 to 21.32 , respectively.

CONCLUSIONS: Estimates of the mean risk of WNV transmission by transfusion ranged from 1.46 to 12.33 per 10,000 donations for six high-incidence metropolitan areas during the 2002 epidemic. Because the risk was highly geographically and temporally variable, computation of geographically localized estimates is recommended. The derived approximating formula for the mean risk performed well for the estimates given.
$\mathrm{W}$ est Nile virus (WNV), a mosquito-borne flavivirus, was first identified in North America in an epizootic among birds and horses and an epidemic of human encephalitis and meningitis in the New York City metropolitan area in the late summer and fall 1999. ${ }^{1}$ Human infection can result in a wide array of outcomes, ranging from asymptomatic to a flu-like febrile illness to encephalitis and meningitis, which may result in death. Cases of human WNV encephalitis and meningitis and of otherWNV disease have been reported in the US each year since the initial 1999 outbreak, during which 62 cases were identified. Over the 4 years following its introduction in New York, WNV spread south through the Atlantic and Gulf coastal states, west into the Ohio Valley and midwest, and on into the Great Plains and Rocky Mountain states. By 2002, WNV was detected in every state east of the continental divide, with many reports of avian, equine, and human illness throughout this region. As of November 30, 2002, more than 3300 cases of WNV-associated human illness with onset dates in 2002 were reported, with 201 deaths from 37 states and the District of Columbia. ${ }^{2}$ This is the largest outbreak of an arboviral disease recorded in the US and the largest outbreak of WNV meningoencephalitis ever recorded..$^{2,3}$

We previously estimated the risk of transfusionassociated WNV transmission from donations collected during the 1999 WNV outbreak in Queens, New York, with

ABBREVIATION: WNV $=$ West Nile virus.

From the Division of Vector-Borne Infectious Diseases, National Center for Infectious Diseases, Centers for Disease Control and Prevention, Fort Collins, Colorado.

Address reprint requests to: Brad Biggerstaff, $\mathrm{PhD}$, Division of Vector-Borne Infectious Diseases, Centers for Disease Control and Prevention, PO Box 2087, Fort Collins, CO 80522-2087; e-mail: bbiggerstaff@cdc.gov.

Received for publication January 29, 2003; revision received March 13, 2003, and accepted April 17, 2003.

TRANSFUSION 2003;43:1007-1017. 
case onset data from the outbreak and historic data on WNV viremia timing and duration in humans. ${ }^{4}$ With statistical resampling methods, the mean risk (per 10,000 donations) of transfusion-associated transmission was estimated for Queens in 1999 as 1.8 (95\% CI, 1.4-2.2) from donations collected over the course of the outbreak, and the maximum risk (per 10,000 donations) was estimated as $2.7(95 \% \mathrm{CI}, 0.9-5.6){ }^{4}$

Subsequentially during the 2002 WNV epidemic, WNV transmission through transplanted organs and blood donation was documented. ${ }^{5}$ Documentation of transfusion-related WNV transmission has raised the question of screening the US blood supply for WNV; quantitative assessment of the risk of transfusion-associated WNV transmission will help determine the potential benefit of this approach. The risk of transfusion-associated transmission of WNV throughout the 2002 epidemic in the US is estimated, and a simple formula to estimate the mean risk during the outbreak given. Because mosquito-borne WNV transmission is highly localized geographically, so that WNV transfusion-associated transfusion risk can vary geographically, we provide estimates for the six states with the highest incidence of reported
WNV meningoencephalitis (meningitis, encephalitis, or both) and for six high-incidence metropolitan areas. A map of county-level incidences of WNV meningoencephalitis in the 48 contiguous US states and the District of Columbia is shown in Fig. 1. Table 1 lists the states and metropolitan areas for which estimates were computed along with summary statistics of the 2002 outbreak in these areas.

\section{MATERIALS AND METHODS}

\section{General approach}

We estimated the risk of transfusion-associated WNV transmission from reported cases of WNV meningoencephalitis with their dates of symptom onset during the 2002 epidemic along with historic data on the timing and duration of viremia in humans. ${ }^{6}$ Statistical resampling of these data was used to estimate at any time $t$ during the outbreak the number of persons in the population of interest who were viremic and asymptomatic, although for persons who developed symptoms, only the time of viremia before symptom onset was included. This provides a curve, indexed by time $t$, which estimates the

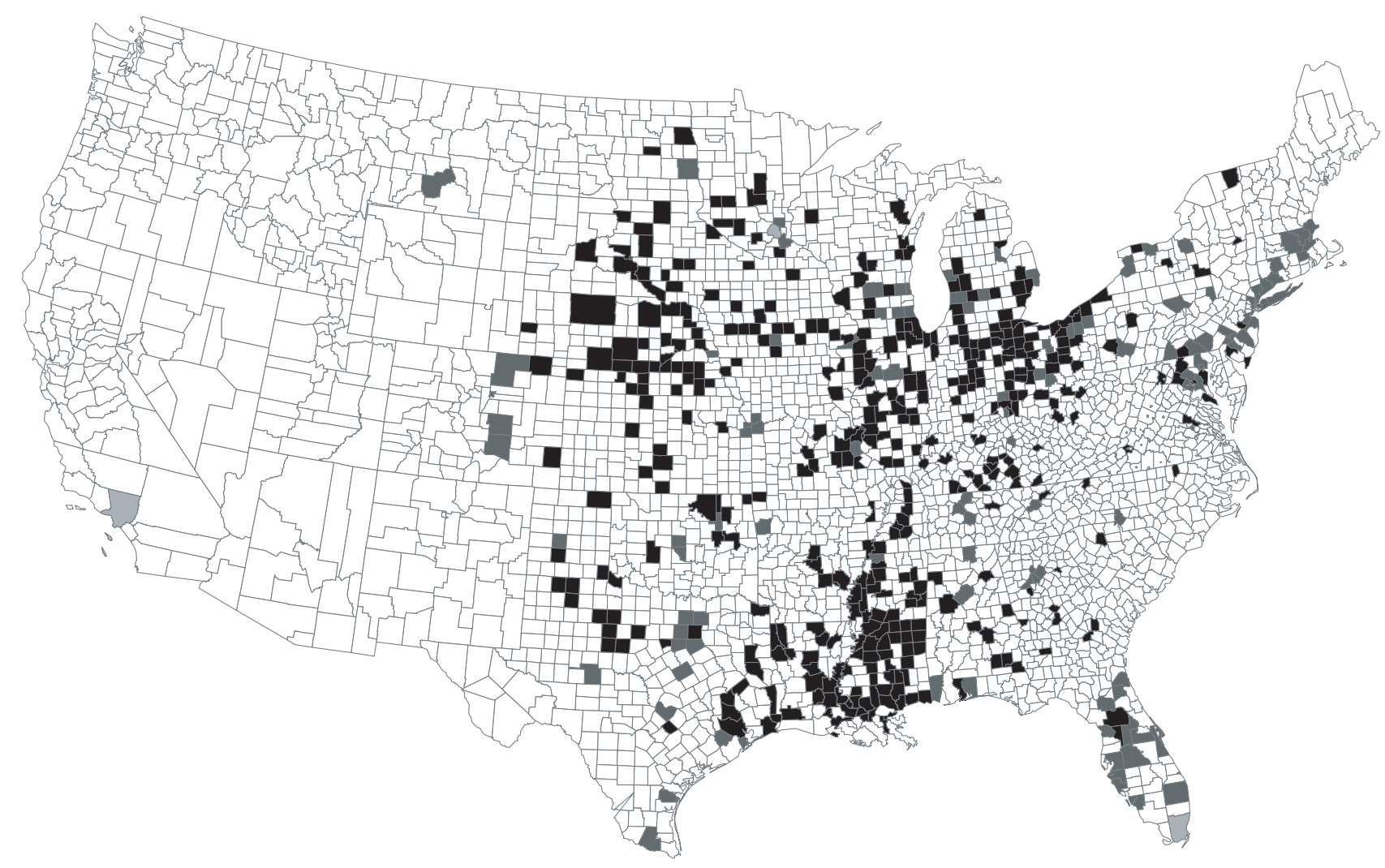

Fig. 1. Incidence of reported cases of WNV meningoencephalitis per 100,000 population by county, 2002. Data are as of January 21, 2003, using population data from the 2000 US Census (http://www.census.gov). Incidence per 100,000: $(\square) 0$; $(\square)>0$ to 0.09 ; ( $\square$ ) 0.1 to 0.9 ; (口) $1+$. 
TABLE 1. Regions for which WNV transfusion risk estimates are computed, with summary WNV case counts and incidence*

\begin{tabular}{|c|c|c|c|c|c|c|}
\hline Region & First case & Last case & Outbreak length (days) & Number of cases & Population & Incidence (per 100,000) \\
\hline \multicolumn{7}{|l|}{ State } \\
\hline Illinois & July 10 & October 13 & 95 & 524 & $12,419,293$ & 4.22 \\
\hline Louisiana & June 10 & November 22 & 165 & 205 & 4,468,976 & 4.59 \\
\hline Michigan & July 20 & October 25 & 97 & 500 & $9,938,444$ & 5.03 \\
\hline Mississippi & June 24 & December 14 & 173 & 157 & $2,844,658$ & 5.52 \\
\hline Nebraska & August 4 & September 30 & 57 & 65 & $1,711,263$ & 3.80 \\
\hline Ohio & July 27 & October 13 & 78 & 296 & 754,844 & 2.61 \\
\hline \multicolumn{7}{|l|}{ Metropolitan area† } \\
\hline Chicago, IL & July 10 & October 1 & 83 & 409 & $6,096,533$ & 6.71 \\
\hline Cleveland, $\mathrm{OH}$ & July 30 & October 3 & 65 & 146 & $1,363,264$ & 10.71 \\
\hline Detroit, MI & July 29 & October 25 & 88 & 414 & $4,080,742$ & 10.15 \\
\hline Houston, TX & July 4 & November 24 & 143 & 95 & $3,400,578$ & 2.79 \\
\hline Memphis, TN & July 30 & September 19 & 51 & 33 & 826,330 & 3.99 \\
\hline St. Louis, MO/IL & July 14 & October 10 & 88 & 92 & $1,841,507$ & 5.00 \\
\hline
\end{tabular}

* Number of WNV cases and incidence is of meningoencephalitis only. Population is from the 2000 US. Census (http://www.census.gov).

† Metropolitan area as defined by counties: Chicago (Cook and Du Page); Cleveland (Cuyahoga); Detroit (Wayne, Oakland, Macomb);

Houston (Harris); Memphis (Shelby); St. Louis (St. Louis, St. Louis City, MO; Madison, St. Clair, IL).

number of potential donors who may transmit WNV through donation over time. We then divided this curve by the population size to estimate the risk of transfusionassociated WNV transmission. Throughout the remainder of the article, the term "case" is used to mean a case of WNV meningoencephalitis.

\section{Study population and data}

The populations of interest were the inhabitants of the regions listed in Table 1 during the 2002 WNV outbreak. Symptom onset dates were used for the cases of WNV meningoencephalitis from the 2002 outbreak as reported by state health departments to and compiled by the Division of Vector-Borne Infectious Diseases, National Center for Infectious Diseases, CDC (Fort Collins, CO). All estimates given are based on reports of WNV meningoencephalitis cases through January 21, 2003.

Figures 2 and 3 show pin plots of the symptom onset dates of the cases of WNV meningoencephalitis for the regions listed in Table 1 during 2002 reported to the CDC through January 21, 2003. Illinois and Michigan reported the largest numbers of cases, with the corresponding largest numbers of cases from the metropolitan areas of Chicago and Detroit. Apparent in these graphs is that the outbreak started earlier and lasted longer in the southern states (Louisiana and Mississippi).

\section{Historic data on WNV viremia distribution}

We supplemented the observed case symptom onset data with historic data from which an assumed distribution for the duration from viremia initiation to symptom onset is derived and from an experimental study by Southam and Moore $^{6}$ on the duration of WNV viremia.
The time from WNV inoculation to symptom onset, the incubation period, is not precisely known but has been reported to be approximately 2 to 6 days. ${ }^{7-9}$ Because there is roughly a 1- to 2-day lag between inoculation with WNV and its detection in the blood, it is assumed that the duration from initiation of viremia to symptom onset is approximately 1 to 5 days. ${ }^{7}$

In a study of human WNV infection, Southam and Moore $^{6}$ give data in their Fig. 4 on the duration of viremia in cancer patients experimentally infected with WNV. The patients were stratified by severity of WNV-associated illness, and those data from patients with demonstrable viremia who displayed either no or mild symptoms were used, because these persons are most representative of WNV-infected persons unlikely to self-defer from donation. These 50 patients had mean viremia duration of 6.3 days with a SD of 2.7 days and median viremia duration of 6.5 days with a range of 1 to 11 days.

\section{Statistical analysis}

As detailed below, a statistical resampling approach was used to estimate for any time $t$ during the outbreak the number of infected persons in the population of interest who were viremic and asymptomatic, excluding the time after symptom onset for persons who developed symptoms, and we then divided this estimate by the population size to yield an estimate of the risk of transfusionassociated WNV transmission at time $t$. This computation differs slightly from the method used for the Queens 1999 outbreak ${ }^{4}$ in that we now account both for infected and for viremic persons who remain asymptomatic and for persons who are asymptomatic at time $t$ but who later develop symptoms. 


\section{Illinois}

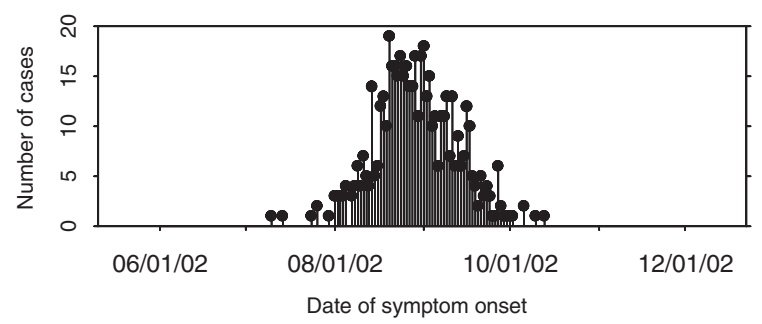

Michigan

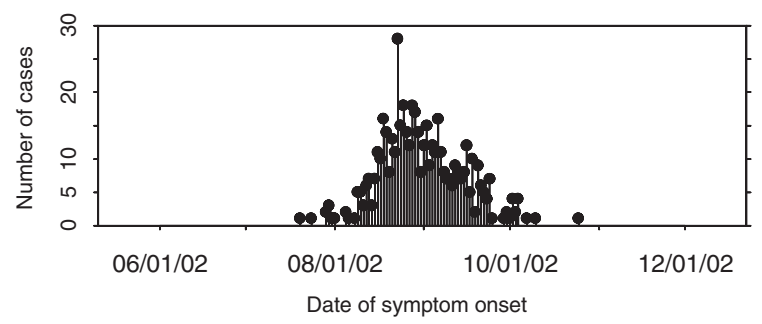

Nebraska

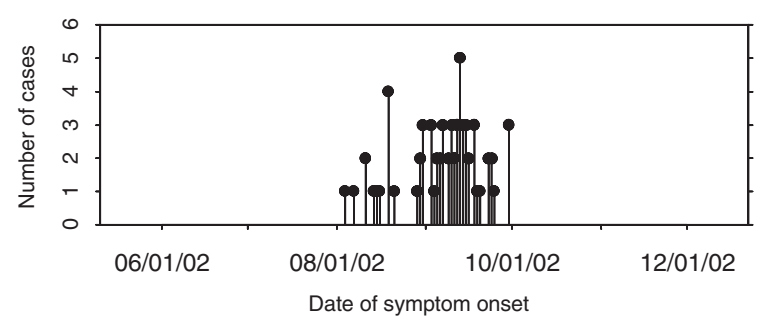

Louisiana

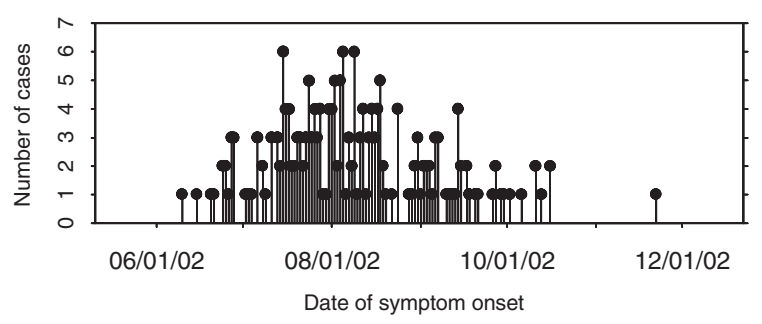

Mississippi

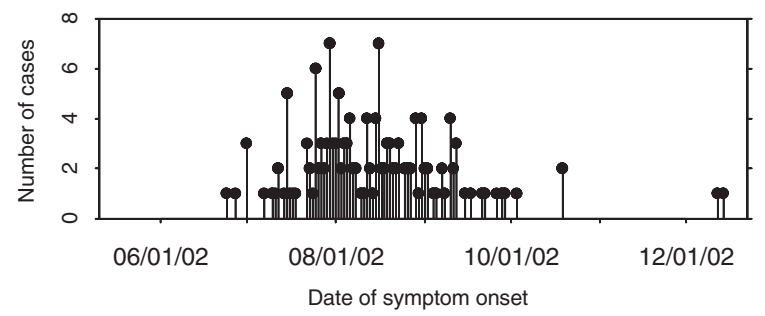

Ohio

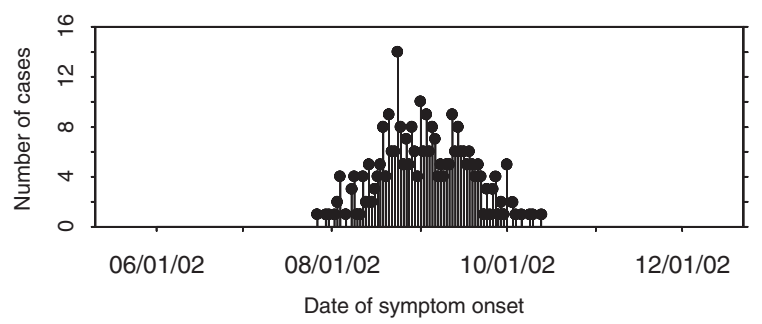

Fig. 2. Number of persons with WNV meningoencephalitis in high-incidence states, reported as of January 21, 2003, by symptom onset date. Note that the individual panels use different scales for the vertical axes.

One-thousand replications of the simulated risk curve, indexed by time $t$, were averaged to produce a final risk curve estimate. Each replication was computed by viewing the case symptom onset times as anchor times and then simulating viremia times for each case based on the exogenous information on viremia timing and duration. Because the underlying $\mathrm{WNV}$ infection process is continuous, but the case onset data are recorded as discrete (in days), the first step in the estimation procedure was to smooth the case onset times by adding an independent smoothing component. To simulate a viremia time for a case, we sampled from an assumed distribution ${ }^{4}$ for the duration from onset of viremia to symptom onset and then subtracted this duration from the smoothed case onset time to represent the time of viremia onset for the case. We next sampled a viremia duration from the Southam and Moore ${ }^{6}$ data and added this to the simulated viremia onset time; the resulting time represented the end of the viremia duration for the case. This procedure was repeated for each case. At this point, then, we have for each case a simulated viremia time or duration, expressed as start and end times of viremia.
It was estimated in the WNV seroepidemiologic survey conducted during the 1999 Queens outbreak that for each case of WNV meningoencephalitis there are 140 WNV-infected persons in the population. ${ }^{10}$ In our computations, therefore, each case's simulated viremia time represents 140 persons' viremia times in the population, and each of the case's simulated viremia times was thus replicated 140 times. It was further estimated in the same study that 21 percent of people infected with WNV develop at least one symptom as a result of the WNV infection, while 79 percent of the WNV-infected people remain asymptomatic. Therefore, in our computations, a random sample of 21 percent of the replicated, simulated viremia times represent symptomatic persons; included for these are only the time before they developed symptoms (and so were potential donors) by truncating these randomly sampled simulated viremia times at the time of symptom onset. For each time $t$ during the outbreak, the number of the resulting simulated durations that contain $t$ were computed to find the estimate for that replication of the number of asymptomatic, viremic persons in the population at time $t$. 


\section{Chicago}

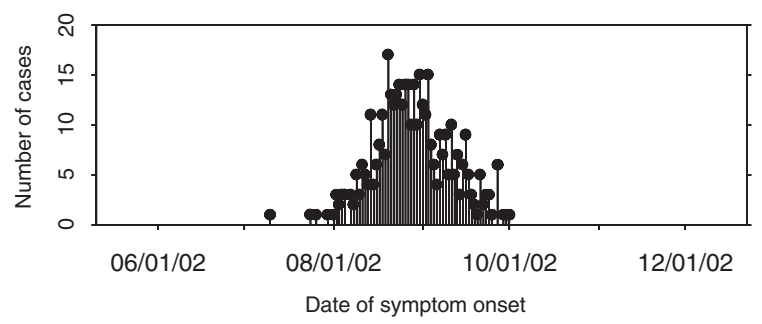

Detroit

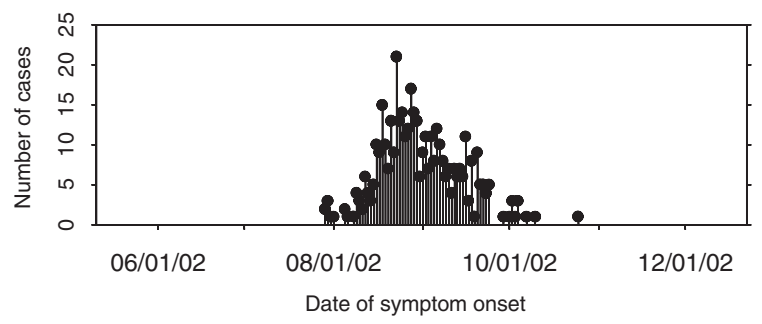

Memphis

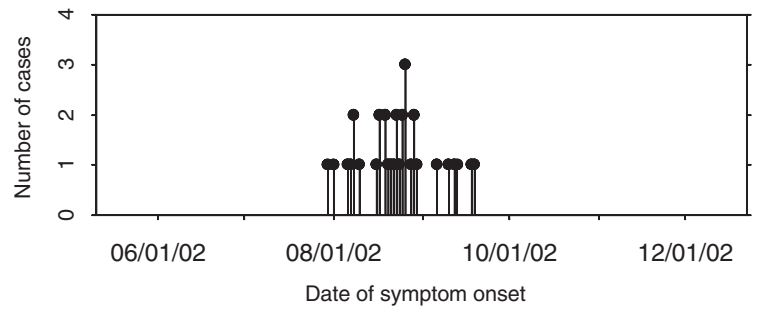

Cleveland

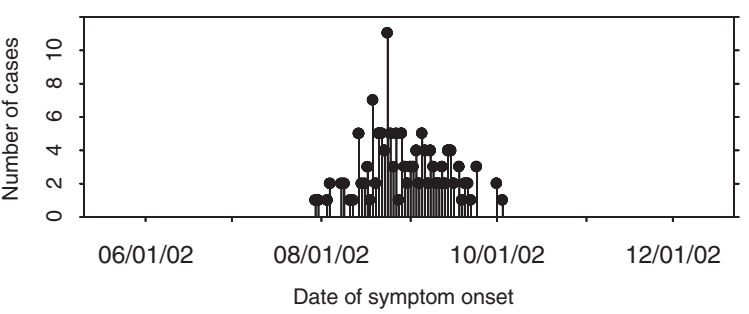

Houston

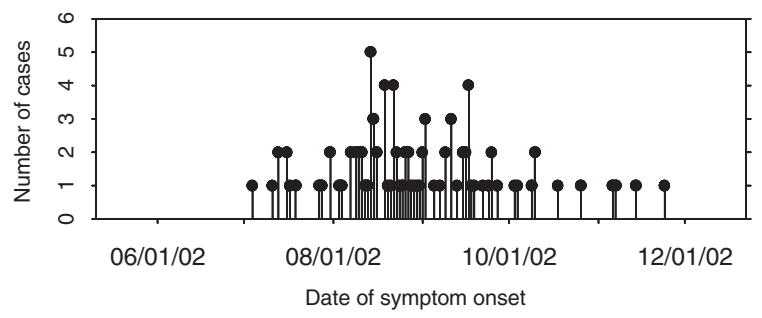

St. Louis

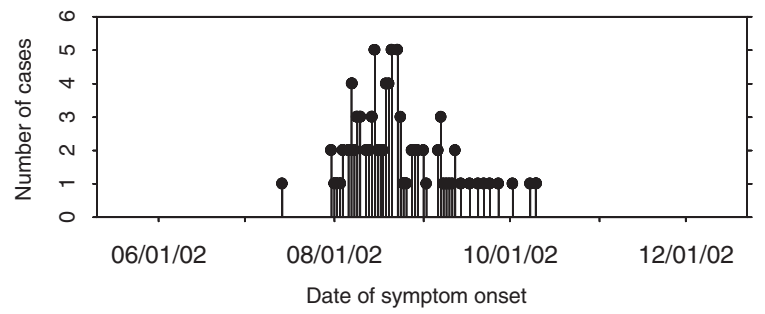

Fig. 3. Number of persons with WNV meningoencephalitis in high-incidence metropolitan areas, reported as of January 21, 2003, by symptom onset date. Note that the individual panels use different scales for the vertical axes.

Finally, we averaged all of the 1000 replicated viremia curves and divided by the population size to yield an estimated risk curve, which is a curve indexed by time $t$ that represents the risk of transfusion-associated WNV transmission over the course of the outbreak. Simultaneous confidence bands for the true number of cases viremic at any given time during the outbreak are computed based on this computation with the percentile- $t$ approach of Cowling et al. ${ }^{4,11}$ The mean and maximum risks (and associated CIs) over the course of the outbreak provide convenient summaries of the full risk curve, so these are also computed. A detailed mathematical development of this approach can be found in Biggerstaff and Petersen, ${ }^{4}$ although slight modifications to that development would be needed to account for the minor adjustments made here.

When inferring to "the population," which varies here as defined by the regions given in Table 1, the following assumptions are made. First, the dates of infection of the cases with known symptom onset dates are similar to those of all residents of the area of interest who became infected. Next, it is assumed that the ratio of the number of WNV meningoencephalitis cases to the number of asymptomatic infections is constant across time, as is the size of the population at risk. Finally, it is assumed that the rate of blood donation is constant over the area and time of interest and that potential blood donors have the same risk of infection with WNV as the general population of the area of interest.

Because of the method used to compute the mean risk of transfusion-associated WNV transmission, it is expected that incidence, $I$, of $\mathrm{WNV}$ meningoencephalitis cases and the mean risk of transfusion transmission are closely related. For each WNV meningoencephalitis case, we assumed 140 infected persons in the population, 79 percent of whom are expected not to develop symptoms from the infection. Each of these persons is on average viremic for 6.3 days, and each of the persons who develop symptoms is viremic a mean of 3 days before developing symptoms. Combining this with the length of the outbreak, $L$, gives the proportion of time an individual is viremic during the outbreak; that is, it provides an estimate of the probability that that person donates blood while viremic. We therefore have the following simple 
formula for mean risk (per 10,000 donations) based on $I$ and $L$, where a scaling factor of 0.1 is included because $I$ is reported per 100,000, while the mean risk of transfusion transmission is reported per 10,000:

$$
\begin{aligned}
\text { Average Risk } & \approx \frac{140 \times 0.79 \times 6.3+140 \times 0.21 \times 3}{L} \times 0.1 \times I \\
& \approx \frac{78}{L} \times I .
\end{aligned}
$$

CIs for the mean risk based on this approximation may be computed with standard CIs for the incidence and multiplying the result by $78 / \mathrm{L}$.

All computations were performed with computer software (S-Plus 6.1 Professional for Windows, Insightful Corp., Seattle, WA) with existing routines or routines written by the authors. (The use of S-Plus is not to be construed as an endorsement of the product by the authors.)

\section{RESULTS}

For the states listed in Table 1, the estimated maximum risk of transfusion-associated WNV transmission ranged from 4.34 to 10.46 per 10,000 donations (Fig. 4), while the mean risks ranged from 2.12 to 4.76 per 10,000 donations. Maximum risk estimates ranged from 3.02 to 21.32 per 10,000 donations for the metropolitan areas listed in Table 1 (Fig. 5), while mean risk estimates for these areas ranged from 1.46 to 12.33 per 10,000 donations. The summary estimates and associated CIs for each area are reported in Table 2.

The plots in Figs. 4 and 5 of the estimated risk curves for the states and metropolitan areas provide detailed information for each location. As expected, the curves follow the general trends of the case reporting data shown in Figs. 2 and 3. Also as anticipated, fewer reported cases result in greater uncertainty, evidenced in Figs. 6 and 7 by the wider confidence bands for the states and metropolitan areas reporting fewer cases.

To facilitate direct comparison among the state and the metropolitan area estimates, just the estimated risk curves themselves are plotted, without confidence bands, in graphs shown in Figs. 6 and 7. In Fig. 6 it can be seen that Louisiana's estimated risk curve is long but relatively low, while Michigan's is short and noticeably peaked.

\section{Illinois}

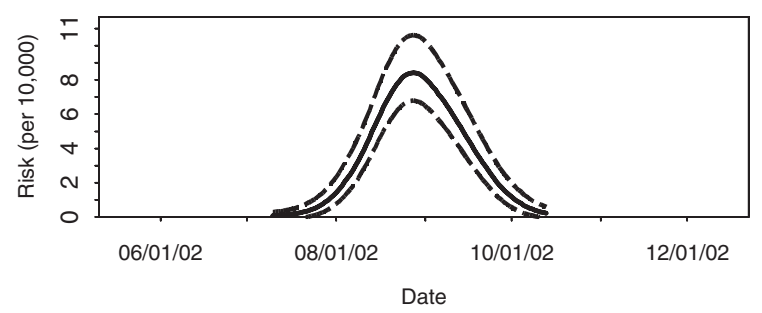

Michigan

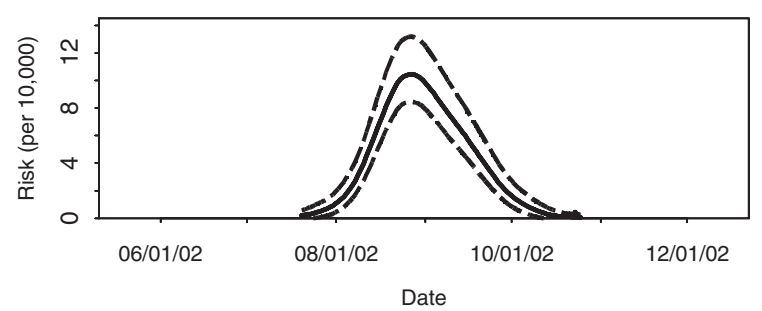

Nebraska

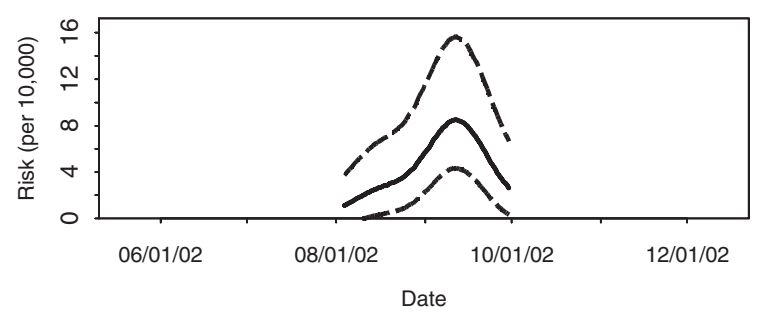

\section{Louisiana}

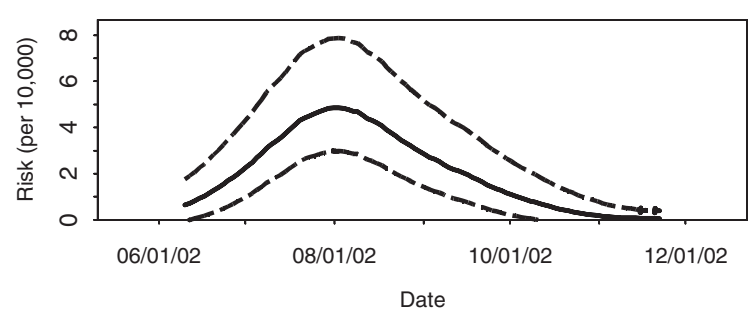

Mississippi

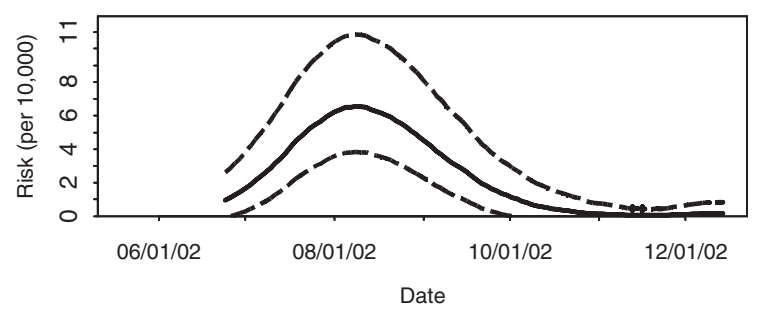

Ohio

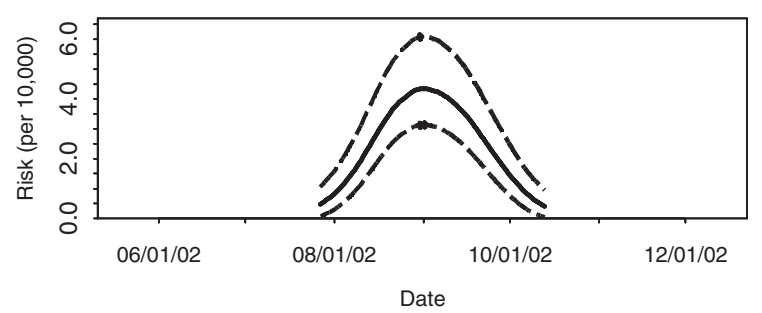

Fig. 4. The estimated transfusion-associated WNV transfusion risk curve (solid, dark line) for the states given in Table 1. Estimates are from 1000 simulations. Simultaneous 95th percentile- $t$ confidence bands are shown as dashed lines. 
Chicago

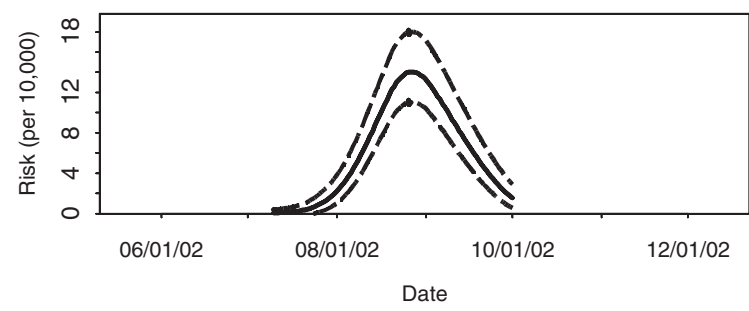

Detroit

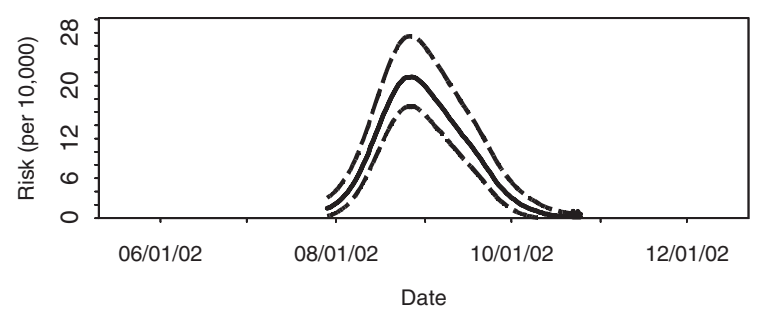

Memphis

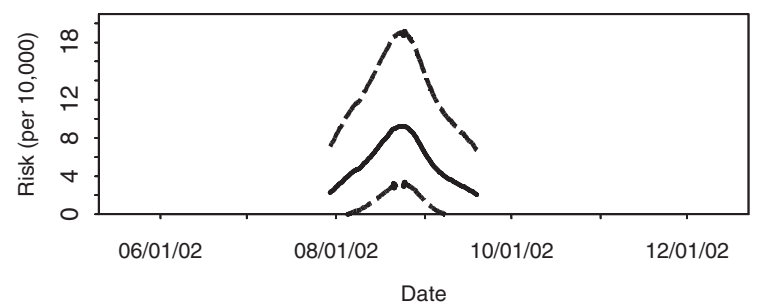

Cleveland

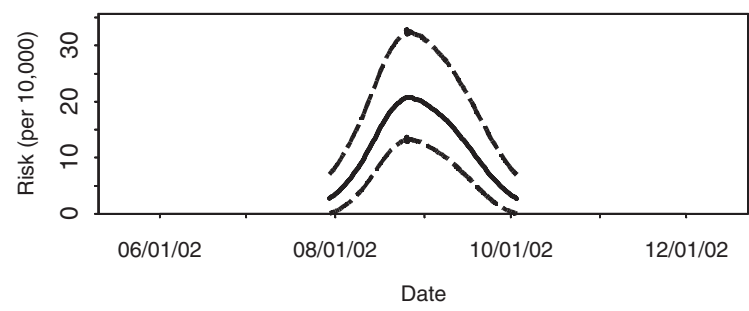

Houston

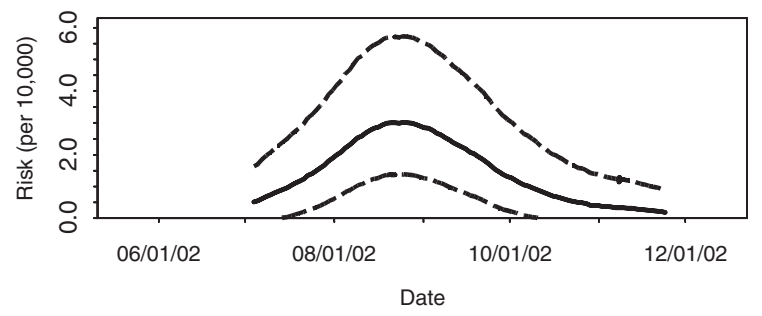

St. Louis

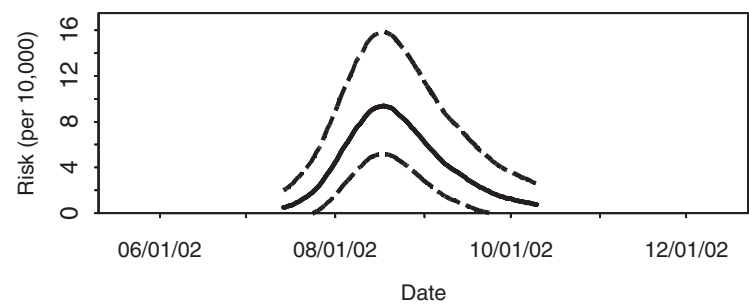

Fig. 5. The estimated transfusion-associated WNV transfusion risk curves (solid, dark lines) for the metropolitan areas given in Table 1. Estimates are from 1000 simulations. Simultaneous 95th percentile- $t$ confidence bands are shown as dashed lines.

TABLE 2. Summary estimates of WNV transfusion risk based on the estimated risk curve and the approximation to the mean risk given in Eq. (1)*

\begin{tabular}{|c|c|c|c|c|c|c|}
\hline Region & Average risk & $95 \% \mathrm{Cl}$ & Maximum risk & $95 \% \mathrm{Cl}$ & Approximate average risk & $95 \% \mathrm{Cl}$ \\
\hline \multicolumn{7}{|l|}{ State } \\
\hline Illinois & 3.44 & $3.32-3.56$ & 8.41 & $6.78-10.61$ & 3.46 & 3.18-3.77 \\
\hline Louisiana & 2.12 & $1.99-2.25$ & 4.86 & $2.98-7.88$ & 2.17 & $1.89-2.49$ \\
\hline Michigan & 4.03 & $3.89-4.17$ & 10.46 & $8.48-13.20$ & 4.05 & $3.71-4.42$ \\
\hline Mississippi & 2.41 & $2.25-2.58$ & 6.56 & $3.84-10.85$ & 2.49 & $2.13-2.91$ \\
\hline Nebraska & 4.76 & $4.17-5.36$ & 8.51 & $4.35-15.66$ & 5.20 & $4.08-6.62$ \\
\hline Ohio & 2.54 & $2.41-2.66$ & 4.34 & $3.14-6.08$ & 2.61 & 2.33-2.92 \\
\hline \multicolumn{7}{|l|}{ Metropolitan area† } \\
\hline Chicago, IL & 6.18 & $5.94-6.42$ & 14.00 & $11.03-17.98$ & 6.30 & $5.72-6.95$ \\
\hline Cleveland, $\mathrm{OH}$ & 12.33 & $11.38-13.28$ & 20.77 & $13.28-32.44$ & 12.85 & $10.93-15.11$ \\
\hline Detroit, MI & 8.89 & $8.53-9.25$ & 21.32 & $16.84-27.45$ & 8.99 & $8.17-9.90$ \\
\hline Houston, TX & 1.46 & $1.33-1.59$ & 3.02 & $1.37-5.37$ & 1.52 & $1.25-1.86$ \\
\hline Memphis, TN & 5.47 & $4.37-6.57$ & 9.23 & $3.18-19.02$ & 6.11 & $4.35-8.58$ \\
\hline St. Louis, MO/IL & 4.33 & $3.95-4.70$ & 9.40 & $5.23-15.87$ & 4.43 & $3.61-5.43$ \\
\hline
\end{tabular}

${ }^{*}$ Estimates are reported per 10,000 donated units. Outbreak durations vary by location and are reported in Table 1.

† Metropolitan area as defined by counties: Chicago (Cook and Du Page); Cleveland (Cuyahoga); Detroit (Wayne, Oakland, Macomb); Houston (Harris); Memphis (Shelby); St. Louis (St. Louis, St. Louis City, MO; Madison, St. Clair, IL). 


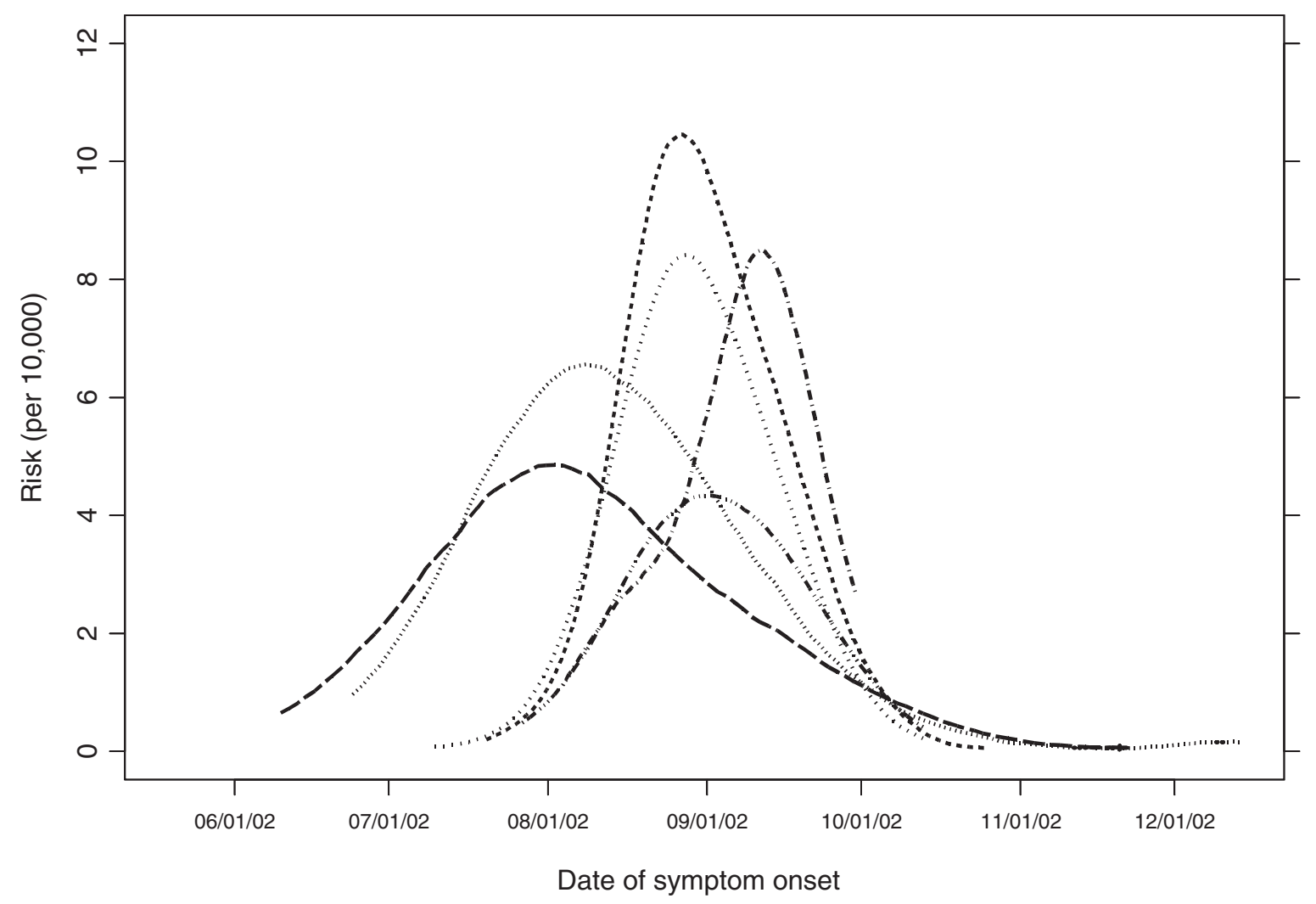

Fig. 6. Estimated transfusion-associated WNV transfusion risk curves for each state in Table 1. ( $\ldots$....) Illinois; ( - - - Louisiana; (- - -) Michigan; ( $(\cdots . .$.$) Mississippi; (.....-) Nebraska; (-.......) Ohio.$

Cleveland and Detroit have nearly identical estimated risk curves (Fig. 7).

We can verify this relationship between the mean risk as computed with the resampling and the full risk curve and the approximating formula given in Eq. (1) for the data set at hand by plotting, as in Fig. $8,(78 / L) \times I$ against the estimated mean risk for each region reported in Table 2. The approximating values computed with Eq. (1) are listed in Table 2. If the approximation is good, the points should follow a straight line with slope 1 . As seen in the graph, the points do appear to lie near the equality line, although two points, representing Memphis and Nebraska, have slightly higher approximating values for the mean risk than computed from the estimated risk curve. Nevertheless, the easily computed formula in Eq. (1) appears to be a reasonable approximation to the mean risk of transfusion over the course of an outbreak. CIs for the approximate mean risks are also reported in Table 2, where the score CI has been used. These approximating CIs are wider than those computed with the resampling method from the estimation of the risk curve.

\section{DISCUSSION}

The WNV epidemic varied considerably geographically, and the corresponding estimated transfusion-associated
WNV transfusion risk from units collected in any particular area also varied. Among the analyzed metropolitan areas, the mean risk was as high as 12.33 per 10,000 donations in Cleveland, with a maximum risk of as high as 21.32 per 10,000 donations in Detroit. These risks are high compared to the estimated risks for other blood-borne pathogens in the US blood supply. In particular, the estimates of mean risk given are nearly 2000 times as high as than those reported for the regularly screened pathogens HCV and HIV, and they are some 200 times as high as that for HBV. ${ }^{12}$ Compared to these other pathogens, however, the risk for transfusion-associated transmission of WNV is highly temporally and spatially limited. Furthermore, the estimates given are during a large epidemic, during which natural transmission of the virus is highest.

WNV transmission from infected RBCs, PLTs, and FFP has been documented and development ${ }^{5}$ of NATs suitable for WNV screening of the US blood supply is now under way (http://www.fda.gov/cber/safety/westnile.htm). The cost-effectiveness of WNV nucleic acid screening will largely depend on the incidence of WNV infection among potential blood donors. Compared to other pathogens screened in the US blood supply, WNV has a very short duration in blood and its incidence in the US to date is highly variable, both geographically and temporally. The cost-effectiveness of screening is therefore likely to vary 


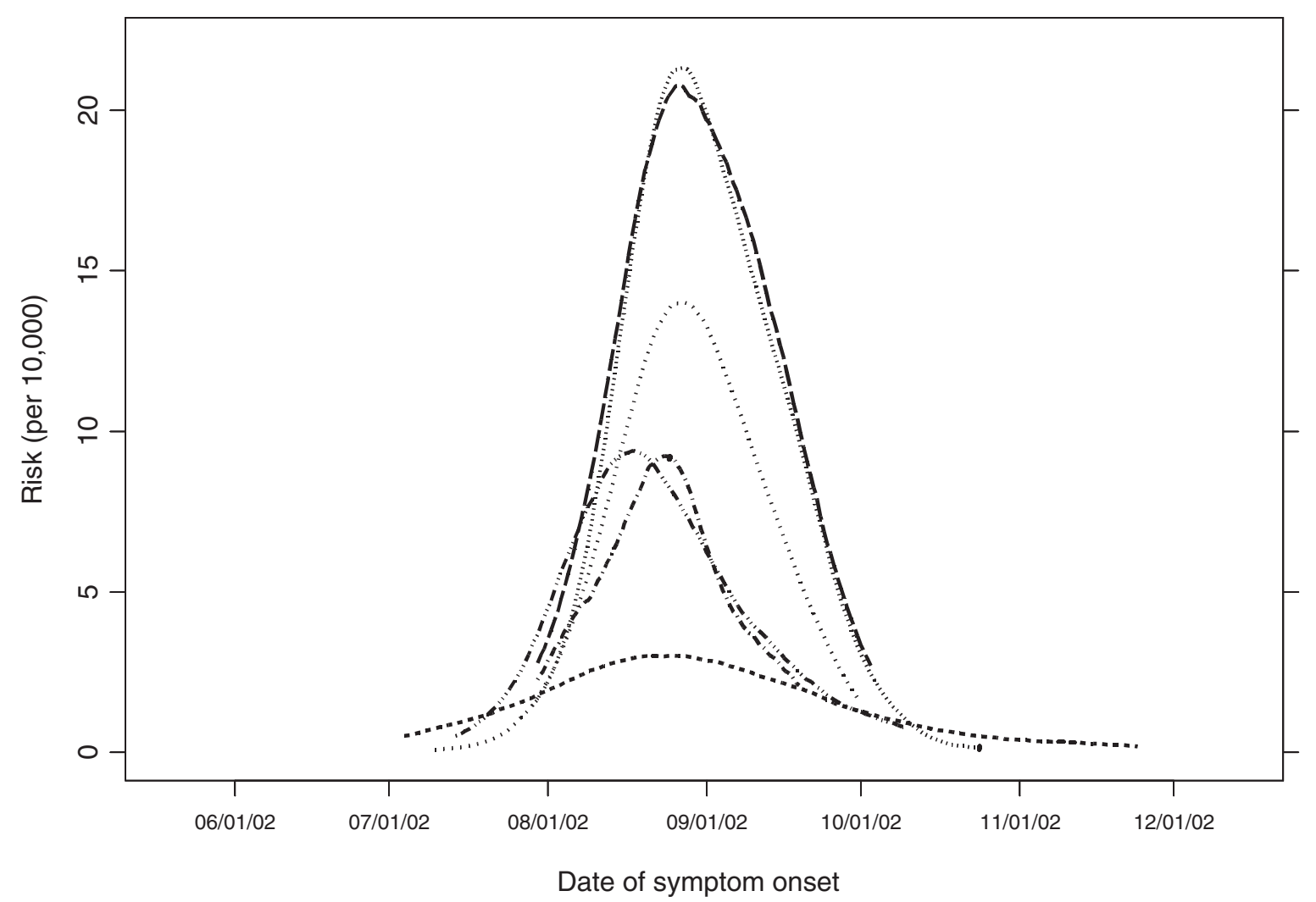

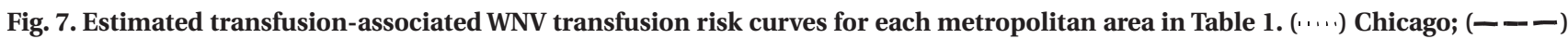
Cleveland; ( ......) Detroit; (- - -) Houston; (.....-) Memphis; (-........) St. Louis.

greatly by year, season, and location; thus, its overall benefit cannot be fully determined for years to come.

Our data have several limitations, and potential, unaccounted-for biases may impact our results. The risk estimates given may overestimate the true transfusionassociated WNV transmission risk for several reasons. One is that the proportion of potential viremic blood donors who would self-defer or be excluded because of symptoms is unknown. As our estimates incorporate, most infected persons remain asymptomatic, however, and many symptomatic donors would have symptom onset after donation, thus limiting the potential impact of symptom-based self-deferral or exclusion. One-hundred percent transmission of virus from viremic donors is assumed, and the true value is probably less. Our estimates can easily be adjusted to account for this by multiplying them by the presumed rate of such transmission. Uncertainties about the duration of viremia persist, given that the data used for the duration of viremia were from experimental infections of cancer patients. The duration of WNV viremia in cancer patients may, for example, be longer than for previously healthy persons.

Little is known about the comparative levels of viremia among asymptomatic persons infected with WNV and among those who develop symptoms. Of the first 14 identified donors associated with transfusion-related WNV transmission to recipients, 3 had symptom onset in the days before donation, 5 had symptom onset in the days after donation, 1 had symptom onset the day of donation, and 5 remained asymptomatic (CDC, unpublished data). These data suggest that symptomatic persons may be more likely to transmit WNV infection to blood recipients, perhaps because they have a higher or longer viremia than asymptomatic persons. There is insufficient quantitative or even qualitative information concerning viremia and symptomatality to make reasonable accommodation for it in our method. If asymptomatic but viremic donors were less likely to transmit WNV, our estimates would be biased high.

An interesting aspect of the 14 identified, WNVinfected and viremic donors is that 3 of them developed symptoms before donation. It was assumed in our method that these persons would have been excluded from donation. The proportion of symptomatic and viremic persons from whom donations may for whatever reason be accepted is unknown, so we are unable to account for this in our approach. The effect of this would be to bias our estimates downward, as increasing the number of viremic donors by accepting donations from symptomatic persons would increase the risk of transfusion-associated WNV 


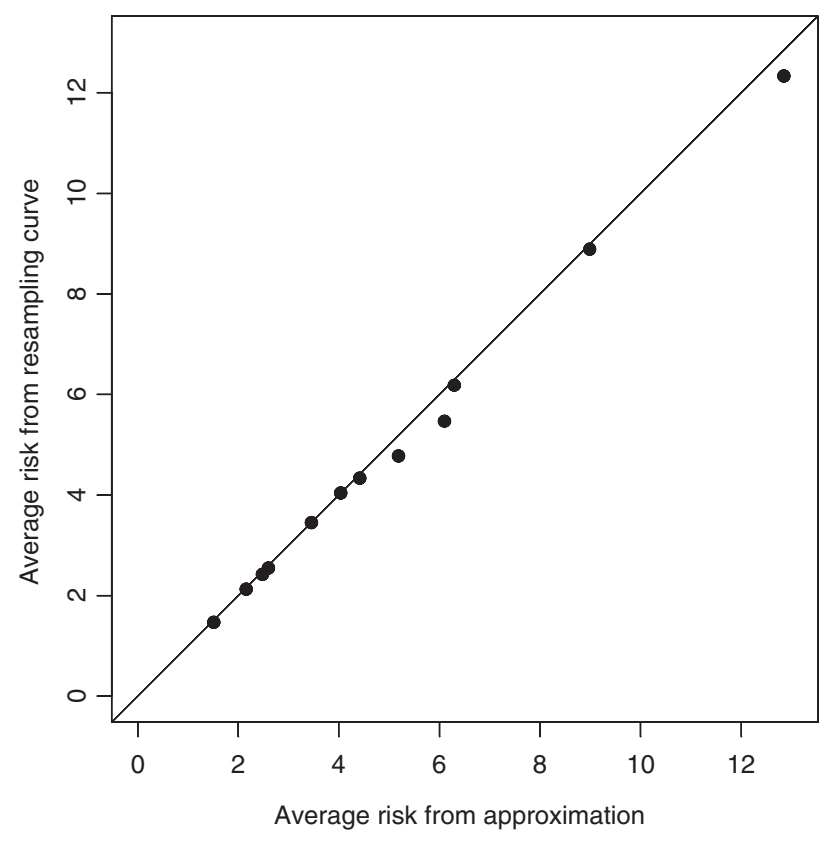

Fig. 8. Mean risk of transfusion-associated WNV transmission calculated with the estimated risk curve (Table 2) versus approximations to the mean risk using the adjusted incidence (Eq. 1). The diagonal line represents perfect agreement between the approximating values and the estimates based on the estimated risk curve.

transmission. Further, our estimates may be too low if case ascertainment by the surveillance system is imperfect.

We computed estimates for regions of the US of dramatically different geographic size, and the appropriateness of the assumptions underpinning the estimates must be taken into consideration when interpreting them. Because the risk of WNV infection is highly localized geographically, it is expected that the prevalence of viremia among potential blood donors will vary greatly over the whole 48 contiguous US states or even over a whole state. Risk estimates can and should therefore be computed for smaller geographic areas for which the constant risk assumption is believed to be more tenable. In our presentation, for example, estimates were provided for the states of Illinois and Michigan, but the estimates for Chicago and Detroit, the metropolitan areas with the highest number of cases in these states, are expected to provide more accurate estimates of local risk for these areas than provided by the statewide estimates.

The approximating formula in Eq. (1) for the mean risk may be most useful for estimating mean transfusionassociated WNV transmission risk when there are too few cases for the computation of the estimated risk curve to be reliable. As an example, the number of reported WNV meningoencephalitis cases for the Baltimore, Maryland, metropolitan area (Baltimore, Baltimore City, and Anne Arundel counties) was nine, spanning over 69 days from
July 27 through October 4, 2002. Using the population value $1,832,567$, derived from 2000 US Census data on the county level, an estimate of the mean risk can be computed as $78 \times(9 \times 100,000 / 1,832,567) / 69=0.56$ per 10,000 donations; the associated score $\mathrm{CI}$ is 0.29 to 1.06 per 10,000 donations.

While the formula in Eq. (1) should provide a useful tool to compute the mean risk, there does not appear to be such a straightforward relation between incidence and the maximum risk. No such relationship should be expected, however, as the maximum risk depends not only on the number of WNV meningoencephalitis cases but also on when they accumulate. Greater detail and information is thus contained in the estimated risk curves plotted in Figs. 4 and 5 than in the estimates of mean risk only. In particular, transfusion-associated WNV transfusion risk can be more than two times as high near the peak of the outbreak than the mean would indicate (see the estimates in Table 2), so that the full risk curve should be utilized when detailed, time-specific estimates of risk are required. Nevertheless, estimation of the mean risk by local or regional agencies who may have only summary incidence and outbreak length information should find the approximating formula in Eq. (1) useful when estimating the impact of WNV activity in their areas.

Our methods are reasonably straightforward and computationally inexpensive, relying only on the availability of case onset dates and execution of computer simulation, but they are indirect. A more direct method of estimating the prevalence of WNV-infected units, and so the risk of transfusion-associated WNV transmission, would be a direct sampling of donated units and subsequent testing of them for the presence of WNV. Given the magnitudes of the prevalence expected based on the computations given in this article, such a direct method of estimation would be expected to require a large number of samples to achieve reasonable precision. Such studies in areas with substantial WNV epidemics would help determine the accuracy of estimates obtained from the calculations presented here. These direct estimates will likely prove essential in evaluating future screening measures for WNV.

\section{ACKNOWLEDGMENTS}

The authors thank Daniel R. O'Leary, DVM, Aaron M. Kipp, and the ArboNet Surveillance System technical staff of the Division of Vector-Borne Infectious Diseases, National Center for Infectious Diseases, CDC, for assistance with obtaining the case onset data.

\section{REFERENCES}

1. Nash D, Mostashari F, Fine A, Miller J, et al. The outbreak of West Nile virus infection in the New York City area in 1999. N Engl J Med 2001;344:1807-14. 
2. Provisional surveillance summary of the West Nile virus epidemic-United States, January-November 2002. MMWR Morb Mortal Wkly Rep 2002;51:1129-33.

3. Campbell GL, Marfin AA, Lanciotti RS, Gubler DJ. West Nile virus. Lancet Infect Dis 2002;2:519-29.

4. Biggerstaff BJ, Petersen LR. Estimated risk of West Nile virus transmission through blood transfusion during an epidemic in Queens, New York City. Transfusion 2002; 42:1019-26.

5. Investigations of West Nile virus infections in recipients of blood transfusions. MMWR Morb Mortal Wkly Rep 2002; 51:973-4.

6. Southam CM, Moore AE. Induced virus infections in man by the Egypt isolates of West Nile virus. Am J Trop Med Hyg 1954;3:19-50.

7. Goldblum N, Sterk VM, Paderski B. The clinical features of the disease and the isolation of West Nile virus from the blood of nine human cases. Am J Hygiene 1954;59:89-103.
8. Kokernot RH, McIntosh BM. Isolation of West Nile virus from a naturally infected human and from a bird, Sylvitta rufescens (Vieillot). S Afr Med J 1959;33: 987-9.

9. Hannoun C, Corniou B, Causse G, Panthier R. Development of serum antibodies in 4 cases of West Nile virus infection. Ann Inst Pasteur (Paris) 1967;113: 29-36.

10. Mostashari F, Bunning ML, Kitsutani PT, et al. Epidemic West Nile encephalitis, New York, 1999: results of a household-based seroepidemiological survey. Lancet 2001; 358:261-4.

11. Cowling A, Hall P, Phillips MJ. Bootstrap confidence regions for the intensity of a Poisson point process. J Am Stat Assoc 1996;91:1516-24.

12. Busch MP, Kleinman SH, Nemo GJ. Current and emerging infectious risks of blood transfusions. JAMA 2003;289: 959-62. 